\title{
Avulsión de parte de una falange, síndrome del túnel carpiano y contractura de Dupuytren. ¿Coincidencia o relación causal?
}

\author{
Avulsion of part of a phalanx, carpal tunnel syndrome \\ and Dupuytren's contracture. Coincidence or causal \\ relationship?
}

\section{A. Ortega Pérez}

\begin{abstract}
RESUMEN
En la mayoría de los casos, el origen del síndrome del túnel carpiano (STC) y el de la contractura de Dupuytren son desconocidos, sin embargo aquí presentamos la asociación de ambos padecimientos en la mano de una enferma que pocos meses antes había sufrido la avulsión de parte de una falange en ella. Al comparar los hechos con los datos de la bibliografía científica, concluimos que la avulsión fue la causa del STC o bien su desencadenante directo en una mujer que tendría alguna predisposición anatómica a padecerlo. La conclusión es aún más clara para la contractura de Dupuytren en estadio inicial en esta lesionada. Ambas enfermedades aparecieron después del traumatismo, su relación causal es coherente con los conocimientos científicos, se han publicado ya casos similares y esta mujer carecía de la mayoría de los factores de riesgo descritos en la bibliografía tanto para el STC como para la contractura palmar idiopáticos.
\end{abstract}

Palabras clave: Contractura de Dupuytren, etiología, postraumático, síndrome del túnel carpiano, traumatología.

\section{ABSTRACT}

In most cases, the etiology of carpal tunnel syndrome (CTS) and of Dupuytren's contracture are unknown. However, here both diseases are presented associated in the hand of a woman that several months before had suffered the avulsion of part of a phalanx. Comparing these facts with the data from the literature, the conclusion is that the avulsion has been either the cause of the CTS or its direct trigger in a woman that probably had some anatomical predisposition for the disease; this conclusion is even clearer for the initial stage of Dupuytren's contracture in this woman, because both appeared after the trauma, its causal relationship is coherent with the scientific knowledge, given that similar situations have been described, and because the woman lacked most of the risk factors associated in the literature with the idiopathic CTS or with the idiopathic Dupuytren's contracture.

Key words: Dupuytren's contracture, etiology, post-traumatic, Carpal tunnel syndrome, traumatology.

Correspondencia: Dr. Arturo Ortega. Unidad de Medicina Legal. Facultad de Medicina y Ciencias de la Salud. C/ Sant Llorenç, 21. 43201 - Reus (Tarragona). Correo electrónico: aop@fmcs.urv.es

${ }^{1}$ Doctor en Medicina. Profesor de Medicina Legal y Toxicología. Facultad de Medicina y Ciencias de la Salud. Universidad "Rovira i Virgili". 


\section{INTRODUCCIÓN:}

Tanto el síndrome del túnel carpiano (STC) como la contractura de Dupuytren (CD) se consideran de origen generalmente desconocido, pero en este artículo presentamos la asociación de ambos padecimientos en la misma mano de una enferma, lo cual en sí ya es una coincidencia llamativa, pero lo más interesante del caso es que esta mujer había sufrido pocos meses antes un traumatismo violento en un dedo de dicha mano.

\section{CASO CLÍNICO:}

Una mujer de cincuenta años sufrió un accidente en el que el dedo anular de su mano izquierda quedó atrapado entre una puerta y su marco, ambos de aluminio. La mujer dio un tirón reflejo de la mano y sufrió la avulsión de la parte atrapada, aproximadamente la mitad distal de la tercera falange de ese dedo, con una fractura conminuta de ese hueso y la conservación de una parte de la matriz ungueal. En el Servicio de Urgencias del hospital le reconstruyeron el muñón bajo anestesia troncular.

Después de las curas locales y de la rehabilitación preceptiva, el dedo quedó con cierta rigidez y con una tendencia a levantarse y a acabalgar sobre el tercero. Estos hechos se atribuyeron a la luxación del tendón del extensor del anular a nivel de la articulación metacarpofalángica y al desgarro de las bandeletas de inserción de los músculos flexores e interóseos de los dedos, lo que provocó la pérdida de función de estos.

Asimismo, a los pocos meses del accidente la mujer había comenzado a quejarse de parestesias y de dolor en los dedos de la mano izquierda, síntomas que se intensificaron paulatinamente. El estudio electromiográfico realizado ocho meses después del accidente los atribuyó tanto a la "denervación sensitiva parcial del cuarto dedo izquierdo por lesión de las ramas cubitales a nivel distal (palma)" como a un "síndrome del túnel carpiano bilateral de grado moderado." Debe señalarse que esa detección electromiográfica de STC en la mano contralateral no se acompañó en ningún momento de parestesias o de dolor en el área.

En la visita de seguimiento llevada a cabo casi nueve meses después del accidente -el tratamiento de la lesión del hueso y de los tejidos blandos había finalizado seis meses antes-, el traumatólogo apreció unos nódulos duros en la palma de la mano izquierda de la mujer, sobre el trayecto de los tendones flexores de los dedos tercero y cuarto. El especialista interpretó esos nódulos palmares como el inicio de una contractura de Dupuytren postraumática.

Quince meses después del accidente intervenían quirúrgicamente a la mujer para liberar el túnel carpiano izquierdo, mientras que continuaba sin padecer molestias atribuibles a un STC en la mano derecha.

La lesionada había trabajado hasta el día del accidente como administrativa en un pequeño comercio y carecía de antecedentes clínicos de signos o síntomas referidos a las extremidades superiores, así como de antecedentes de obesidad, de diabetes ni de hipotiroidismo. Preguntada sobre la cuestión, no recordaba que ninguno de sus familiares próximos hubiera sufrido STC, CD, dedo en gatillo o enfermedad de Peyronie.

\section{DISCUSIÓN:}

De este caso lo que más llama la atención es la coincidencia en la misma lesionada de un STC y de una CD, ambos postraumáticos. Si la mujer hubiera sufrido una sola de estas complicaciones habría sido fútil presentar aquí su caso, dado que en la bibliografía se ha publicado esa relación en suficientes ocasiones, como se verá en los párrafos siguientes, como para descartar el ocupar páginas de la revista con ella. 
El autor no pretende ofrecer una explicación sobre el por qué ocurrieron ambas complicaciones a la vez, tan solo quiere llamar la atención sobre esa coincidencia curiosa. Sin embargo, sí puede sostener la relación causal entre lesión y STC o entre lesión y CD, ya que el caso de esta mujer cumple varios de los criterios de causalidad de Simonin: el plazo entre la lesión y las complicaciones es el esperable y la progresión de las manifestaciones cumple el criterio de 'síntomas puente', dado el período de latencia necesario hasta la detección de las lesiones; esas lesiones no existían antes del traumatismo -veremos ahora si tenía los factores de riesgo comunes para que aparecieran espontáneamente-, y no existe una causa extraña al traumatismo que pudiera haber provocado esas complicaciones.

En cuanto a la relación etiopatogénica entre la avulsión y los síndromes postraumáticos, algún investigador ha propuesto una tenosinovitis ascendente desde una lesión en la palma o en el dedo como causa del STC o bien la presencia, comprobada, de tejido muscular deshilachado en la palma después precisamente de una avulsión, que comprimía el nervio mediano. En cambio, por lo que se refiere a la $C D$, en la revisión bibliográfica realizada por el firmante no ha aparecido ninguna propuesta de explicación para el mecanismo de la CD postraumática.

El origen del STC es el atrapamiento del nervio mediano, lo que se manifiesta como parestesias, dolor, debilidad de las manos y calambre, típicamente en la mano y en la muñeca, pero no siempre restringido a ellas; lo más común es que afecte a uno o más de los tres primeros dedos. El signo de Phalen se observa en algo más de la mitad de los pacientes, mientras que el signo de Tinel lo muestran cerca de un tercio de estos [I].

Los estudios epidemiológicos han permitido averiguar que es una enfermedad relativamente común, ya que afecta a cerca del 1,5\% de los trabajadores estadounidenses [2]. Aunque en la mayoría de los casos su origen es desconocido, se han averiguado algunos factores de riesgo para la enfermedad:

- Una ocupación con movimientos repetitivos de giro y de flexión de la muñeca o de la mano es el principal $[3,4,5]$.

- El grupo racial, ya que es cuatro veces más frecuente en los blancos que en el resto de los grupos raciales.

- El sexo, porque es de dos a cinco veces más frecuente en mujeres que en hombres.

- La labor como usuario de herramientas vibrátiles, que dobla el riesgo.

- La edad, que conlleva un aumento continuo del riesgo [2].

- La obesidad, con un aumento del 8 \% en el riesgo para cada aumento unitario en el índice de masa corporal [6,7].

- Otras afecciones, como la enfermedad previa del aparato locomotor $[7,8]$-en particular la artritis y la artrosis [9]-, el uso de fármacos corticoides -incluso en ausencia de artritis-, el hipotiroidismo y la diabetes [6,9].

Por todo lo anterior, la frase "es una enfermedad típica de las mecanógrafas" es una simplificación y un error, ya que de hecho las ocupaciones con mayor prevalencia de quejas de STC son el servicio de correos, la atención sanitaria, la construcción, la fabricación y el montaje [2].

Por otro lado, en cerca del $10 \%$ de los casos, el STC se presenta después de un traumatismo local [10]; una asociación que se viene publicando desde hace más de treinta años [ I I, I2]. El traumatismo puede haber afectado a la muñeca o a la mano, siendo el mecanismo probable en esta última situación una tenosinovitis ascendente. Entre las lesiones descritas como causa de un STC la más común es la fractura de la epífisis distal del radio, con un $17 \%$ de afectados tres meses des- 
pués de la intervención [13]; aunque en muchos casos la compresión nerviosa se alivia con los meses, una de cada II ó I 2 afectaciones se mantienen estables [9, I 4].

Conocidos estos datos epidemiológicos ipodemos dilucidar si el STC es espontáneo o postraumático? Es cierto que la afectada es una mujer de cincuenta años, que era administrativa y que probablemente cuidaba de su casa, pero todos ellos son factores asociados con la enfermedad y a juicio del firmante, no bastan para inclinar la balanza hacia la casualidad, si los comparamos con los criterios de causalidad que sí cumple esta relación.

Otros agentes causales del STC son contusión de la muñeca [ I 5], la inestabilidad de la articulación radiocubital distal, la fractura intraarticular desplazada del extremo distal del radio [12], las fracturas del unciforme y del piramidal [16] o incluso las lesiones de los ligamentos o de los tendones del túnel mientras la persona sujeta el volante durante un accidente de tráfico [17]. Un autor [18] presentó los casos de 96 personas que habían sufrido contusiones en accidentes de tráico y a quienes las manifestaciones de STC les aparecieron antes de que transcurrieran dos meses desde el hecho.

Hemos dicho que la lesión iniciadora o desencadenante del síndrome del túnel carpiano puede estar también en la mano, habiéndose publicado casos de fractura de metacarpiano o del dedo [I0] e incluso la avulsión del meñique [17].

Aunque el caso que aquí presentamos es muy raro, en la bibliografía aparecen situaciones muy similares a la descrita. La primera se refiere a la bilateralidad electromiográfica sin afectación clínica, algo que ocurrió en cerca de las tres cuartas partes de los pacientes con STC postraumático de la serie de Heim et al. [10]. La segunda se refiere a la avulsión citada del dedo meñique, que causó el desarrollo rápido de un STC; la exploración quirúrgica de la palma reveló que pequeños filetes del tejido muscular desgarrado se habían desplazado y ocupaban el espacio bajo la vaina fibrosa, comprimiendo al nervio mediano [17].

Por lo que se refiere a la contractura palmar de Dupuytren, es mucho más frecuente en el noroeste de Europa que en la cuenca mediterránea -la llaman "la enfermedad vikinga"-. Así, entre los islandeses mayores de 45 años están afectados el $19 \%$ de los varones y el 4,5\% de las mujeres [19]. Otros autores, en cambio, hallaron que esta fibromatosis palmar es hasta siete veces más común en los varones que en las mujeres [20]. El inicio en aquellos ocurre hacia los 55-60 años y en estas, unos diez años después [2 I]; con la edad aumenta la prevalencia en ambos sexos, de modo que en los más ancianos la proporción masculino/femenino tiende a equilibrarse. La enfermedad se hereda de una forma autosómica dominante en muchos casos, pero también existen influencias epigenéticas: es más frecuente en quienes realizan tareas manuales pesadas [22], así como en diabéticos y en epilépticos [20] y en las personas delgadas [19]. Los fumadores y los bebedores sufren contracturas más graves que la media [20]. Algo más de la mitad de los enfermos padecen una afectación bilateral [20].

Después de esta introducción general sobre la enfermedad, centrémonos en los casos secundarios a un traumatismo y veremos que el presentado aquí no es el primero que se atribuye a ese origen. La prevalencia es superior en quienes han sufrido traumatismos claros en la mano [23,24], incluso independientemente de su trabajo manual [22]. Padecerán una contractura palmar de Dupuytren, siempre en un grado leve, cerca del I I \% de los afectados por una fractura de Colles desplazada [13] y se han publicado casos desencadenados por quemaduras en la extremidad superior [25], por incisiones quirúrgicas en la mano [26] y por un traumatismo deportivo [27].

En el caso presentado, el mecanismo de la avulsión permite explicar la patogenia de una afectación mucho más proximal que la punta del dedo: esta quedó atrapada y la mujer dio un tirón 
reflejo que arrancó esa porción del dedo, luxó el tendón del extensor del anular en la articulación metacarpofalángica y desgarró las bandeletas de inserción de los músculos flexores e interóseos de los dedos; estas lesiones demuestran que la afectación por el tirón no se limitó al extremo de la falange distal del cuarto dedo.

Como resumen de los resultados de la búsqueda bibliográfica y su comparación con el caso clínico presentado, la avulsión de parte de una falange puede considerarse el agente causal del STC -o su desencadenante directo- y de la contractura de Dupuytren en estadio inicial en esta lesionada.

\section{BIBLIOGRAFÍA:}

I. Nora DB, Becker J, Ehlers JA, Gomes I. Clinical features of 1039 patients with neurophysiological diagnosis of carpal tunnel syndrome. Clin Neurol Neurosurg 2004;107:64-9.

2. Tanaka S, Wild DK, Seligman PJ, Halperin WE, Behrens VJ, PutzAnderson V. Prevalence and work-relatedness of self-reported carpal tunnel syndrome among U.S. workers: analysis of the Occupational Health Supplement data of 1988 National Health Interview Survey. Am J Ind Med 1995;27:45I-70.

3. Blanc PD, Faucett J, Kennedy J], Cisternas M, Yelin E. Self-reported carpal tunnel syndrome: predictors of work disability from the National Health Interview Survey Occupational Health Supplement. Am J Ind Med 1996;30:362-8.

4. Roquelaure Y, Mechali S, Dano C, Fanello S, Benetti F, Bureau D, Mariel J, Martin YH, Derriennic F, Penneau-Fontbonne D. Occupational and personal risk factors for carpal tunnel syndrome in industrial workers. Scand J Work Environ Health 1997;23:364-9. 5. Tanaka S, Wild DK, Cameron LL, Freund E. Association of occupational and non-occupational risk factors with the prevalence of selfreported carpal tunnel syndrome in a national survey of the working population. Am J Ind Med 1997;32:550-6.

6. Solomon DH, Katz JN, Bohn R, Mogun H, Avorn J. Nonoccupational risk factors for carpal tunnel syndrome. J Gen Intern Med 1999;14:310-4.

7. Ferry S, Hannaford P, Warskyj M, Lewis M, Croft P. Carpal tunnel syndrome: a nested case-control study of risk factors in women. Am J Epidemiol 2000;151:566-74.

8. Nordstrom DL, Vierkant RA, DeStefano F, Layde PM. Risk factors for carpal tunnel syndrome in a general population. Occup Environ Med 1997;54:734-40.

9. Geoghegan JM, Clark DI, Bainbridge LC, Smith C, Hubbard R. Risk factors in carpal tunnel syndrome. J Hand Surg $[\mathrm{Br}]$ 2004;29:315-20.

10. Heim D, Stricker U, Rohrer G. Carpal tunnel syndrome after trauma. Swiss Surg 2002;8:15-20.

II. Browne EZ Jr, Snyder CC. Carpal tunnel syndrome caused by hand injuries. Plast Reconstr Surg 1975;56:41-3.

12. McClain EJ, Wissinger HA. The acute carpal tunnel syndrome: nine case reports. J Trauma 1976;16:75-8.
13. Stewart HD, Innes AR, Burke FD. The hand complications of Colles' fractures. J Hand Surg [Br] 1985;10:103-6.

14. Bruske J, Niedzwiedz Z, Bednarski M, Zyluk A. Acute carpal tunnel syndrome after distal radius fractures--long term results of surgical treatment with decompression and external fixator application. Chir Narzadow Ruchu Ortop Pol 2002;67:47-53.

I5. Mack GR, McPherson SA, Lutz RB. Acute median neuropathy after wrist trauma. The role of emergent carpal tunnel release. Clin Orthop 1994;300: I4I-6.

16. Martinet X, Dhont F, Morel M, Menu F, Kadji O, Malka G. Acute carpal tunnel syndrome. A report of an etiological form not previously described. Chir Main 2001;20:388-90.

17. Haas DC, Nord SG, Bome MP. Carpal tunnel syndrome following automobile collisions. Arch Phys Med Rehabil 1981;62:204-6.

18. Ames EL. Carpal tunnel syndrome and motor vehicle accidents. J Am Osteopath Assoc 1996;96:223-6.

19. Gudmundsson KG, Arngrimsson R, Sigfusson N, Bjornsson A, Jonsson T. Epidemiology of Dupuytren's disease: clinical, serological, and social assessment. The Reykjavik Study. J Clin Epidemiol 2000;53:291-6.

20. Brenner P, Krause-Bergmann A, Van VH. Dupuytren contracture in North Germany. Epidemiological study of 500 cases. Unfallchirurg 2001;104:303-II.

21. Ross DC. Epidemiology of Dupuytren's disease. Hand Clin 1999;15: 53-62, vi.

22. Mikkelsen OA. Dupuytren's disease--the influence of occupation and previous hand injuries. Hand 1978;10:1-8.

23. Zachariae L. Dupuytren's contracture. The aetiological role of trauma. Scand J Plast Reconstr Surg 1971;5:116-9.

24. McFarlane RM. Dupuytren's disease: relation to work and injury. J Hand Surg [Am] 1991;16:775-9.

25. Balakrishnan C, Emanuele JA, Chow IJ. Dupuytren's contracture after burns of the upper extremity. J Burn Care Rehabil 1997;18:245-6. 26. Lanzetta M, Morrison WA. Dupuytren's disease occurring after a surgical injury to the hand. J Hand Surg [Br] 1996;21:48I-3.

27. Connelly TJ. Development of Peyronie's and Dupuytren's diseases in an individual after single episodes of trauma: a case report and review of the literature. J Am Acad Dermatol 1999 Jul;4I(I):I06-8. 